\title{
A Demonstrated Optical Tracker With Scalable Work Area for Head- Mounted Display Systems
}

\author{
Mark Ward ${ }^{\dagger}$, Ronald Azuma, Robert Bennett, Stefan Gottschalk, Henry Fuchs \\ Department of Computer Science \\ Sitterson Hall \\ University of North Carolina \\ Chapel Hill, NC 27599-3175
}

\begin{abstract}
An optoelectronic head-tracking system for head-mounted displays is described. The system features a scalable work area that currently measures $10^{\prime} \times 12^{\prime}$, a measurement update rate of $20-100 \mathrm{~Hz}$ with $20-60 \mathrm{~ms}$ of delay, and a resolution specification of $2 \mathrm{~mm}$ and 0.2 degrees. The sensors consist of four head-mounted imaging devices that view infrared lightemitting diodes (LEDs) mounted in a 10' x 12' grid of modular $2^{\prime}$ x 2 ' suspended ceiling panels. Photogrammetric techniques allow the head's location to be expressed as a function of the known LED positions and their projected images on the sensors. The work area is scaled by simply adding panels to the ceiling's grid. Discontinuities that occurred when changing working sets of LEDs were reduced by carefully managing all error sources, including LED placement tolerances, and by adopting an overdetermined mathematical model for the computation of head position: space resection by collinearity. The working system was demonstrated in the Tomorrow's Realities gallery at the ACM SIGGRAPH '91 conference.
\end{abstract}

CR categories and subject descriptors: I.3.1 [Computer Graphics]: Hardware Architecture - threedimensional displays; I.3.7 [Computer Graphics]: ThreeDimensional Graphics and Realism - Virtual Reality

Additional Key Words and Phrases: Head-mounted displays, head tracking

\section{$1 \quad$ Introduction}

It is generally accepted that deficiencies in accuracy, resolution, update rate, and lag in the measurement of head position can adversely affect the overall performance of a HMD [17][24][25]. Our experience suggests that an additional specification requires more emphasis: range.

\footnotetext{
$\dagger$ Present address: Structural Acoustics, 5801 Lease Lane, Raleigh, NC, 27613. (919) 787-0887
}

Figure 1: The existing system in UNC's graphics laboratory

Most existing HMD trackers were built to support situations that do not require long-range tracking, such as cockpit-like environments where the user is confined to a seat and the range of head motion is limited. But many virtual worlds applications, such as architectural walkthroughs, would benefit from more freedom of movement (Figure 2). Long-range trackers would allow greater areas to be explored naturally, on foot, reducing the need to resort to techniques such as flying or walking on treadmills.

Such techniques of extending range work adequately with closed-view HMDs that completely obscure reality. With seethrough HMDs [9][11], however, the user's visual connection with reality is intact and hybrid applications are possible where physical objects and computer-generated images coexist. In this situation, flying though the model is meaningless. The model is registered to the physical world and one's relationship to both must change simultaneously.

This paper describes the second generation of an optoelectronic head-tracking concept developed at the University of North Carolina at Chapel Hill. In the concept's first generation, the fundamental design parameters were explored and a bench-top prototype was constructed [28]. Building on this success, the second-generation tracker is a 
fully functional prototype that significantly extends the workspace of an HMD wearer.

Figure 2: Walkthrough of Brooks' kitchen design that runs with the tracker. Actual resolution of images seen in the HMD is much lower than this picture's resolution.

The current system (Figure 1) places four outward-looking image sensors on the wearer's head and locates LEDs in a $10^{\prime} \mathrm{x}$ $12^{\prime}$ suspended ceiling structure of modular $2^{\prime} \times 2^{\prime}$ ceiling panels. Each panel houses 32 LEDs, for a total of 960 LEDs in the ceiling. Images of LEDs are formed by lateral-effect photodiode detectors within each head-mounted sensor. The location of each LED's image on a detector, or photocoordinate, is used along with the known LED locations in the ceiling to compute the head's position and orientation. To enhance resolution, the field of view of each sensor is narrow. Thus, as shown in Figures 3 and 7, each sensor sees only a small number of LEDs at any instant. As the user moves about, the working set of visible LEDs changes, making this a cellular head-tracking system.

Measurements of head position and orientation are produced at a rate of $20-100 \mathrm{~Hz}$ with $20-60 \mathrm{~ms}$ of delay. The system's accuracy has not been measured precisely, but the resolution is $2 \mathrm{~mm}$ and 0.2 degrees. It was demonstrated in the Tomorrow's Realities gallery at the ACM SIGGRAPH ' 91 conference, and is, to our knowledge, the first demonstrated scalable head-tracking system for HMDs.

The system is novel for two reasons. First, the sensor configuration is unique. Other optical tracking systems fix the sensors in the environment and mount the LEDs on the moving body [30]. The outward-looking configuration is superior for it improves the system's ability to detect head rotation. The scalable work space is the system's second contribution. If a larger work space is desired, more panels can be easily added to the overhead grid.

\section{Previous work}

Many tracking systems precede this effort, and we will briefly survey representative examples. The essence of the problem is the realtime measurement of the position and orientation of a rigid moving body with respect to an absolute reference frame, a six-degree-of-freedom (6DOF) measurement problem. Solutions are relevant to many other fields.

To our knowledge, four fundamentally different technologies have been used to track HMDs: mechanical, magnetic, ultrasonic, and optical.

The first HMD, built by Ivan Sutherland [27], used a mechanical linkage to measure head position. A commercial product, The Boom [12], uses a mechanical linkage to measure the gaze direction of a hand-held binocular display. The Air Force Human Resources Laboratory (AFHRL) uses a mechanical linkage to measure the position and orientation of a HMD used for simulation [24]. Mechanical systems have sufficient accuracy, resolution, and frequency response, yet their range is severely limited, and a mechanical tether is undesirable for many applications.

Magnetic-based systems [3][21] are the most widely used hand and head trackers today. They are small, relatively inexpensive, and do not have line-of-sight restrictions. Their primary limitations are distortions caused by metal or electromagnetic fields, and limited range [13].

Ultrasonic approaches have also been successful, such as the commercially-available Logitech tracker [20]. Time-of-flight measurements are used to triangulate the positions of sensors mounted on the HMD. The strength of this technology is minimum helmet weight [13]. Physical obscuration as well as reflections and variations of the speed of sound due to changes in the ambient air density make it difficult to maintain accuracy [5].

Because of the potential for operation over greater distances, optical approaches are plentiful, and it is helpful to categorize them on the basis of the light source used. Visible, infrared, and laser light sources have each been exploited.

Ferrin [13] reports the existence of a prototype helmet tracking system using visible light. Although it only tracks orientation, it is worth mentioning here because of its unique approach. A patterned target is placed on the helmet and a cockpit-mounted video camera acquires images in real time. The pattern is designed to produce a unique image for any possible head orientation. The strength of this approach is the use of passive targets which minimize helmet weight. Reflections and other light sources are potential sources of error.

Bishop's Self-Tracker [7] is a research effort involving visible light. A Self-Tracker chip senses incremental displacements and rotations by imaging an unstructured scene. A headmounted cluster of these chips provide sufficient information for the computation of head position and orientation. Although still under development, the concept is mentioned here because it would allow an optical tracking system to operate outdoors, where a structured environment, such as our ceiling of LEDs, would be impossible to realize. 
Because of the difficulties associated with processing information in an unstructured scene, most high-speed optical measurement systems use highly-structured infrared or laser light sources in conjunction with solid-state sensors. The sensor is a often a lateral-effect photodiode as opposed to a true imaging device, because the photodiode produces currents that are directly related to the location of a light spot's centroid on its sensitive surface [32]. The resultant sensor is relatively insensitive to focus, and the light spot's location, or photocoordinate, is immediately available without the need for image processing.

During the 1970's, Selspot [23] popularized the use of infrared LEDs as targets and lateral-effect photodiodes as sensors in a commercially-available system. Their primary emphasis was, and still is, on the three-dimensional locations of individual targets. That is, the Selspot system does not automate the computation of a rigid body's orientation. In a response to this shortcoming, Antonsson [2] refined the Selspot system for use in dynamic measurements of mechanical systems. The resultant system uses two Selspot cameras to view a moving body instrumented with LEDs. Similar approaches have been applied to HMD systems in cockpits [13] and in simulators [11].

The use of an LED light source limits the range of these systems. Typically, the distance between source and detector can be no greater than several feet. Longer distances can be spanned with laser light sources.

The only known example of a 6DOF tracker using laser sources is the Minnesota Scanner [26]. With this sytem, scanning mirrors are used to sweep orthogonal stripes of light across the working volume. Photodiodes are both fixed in space and placed on the moving body. By measuring the time between a light stripe's contact with a fixed and moving photodiode, the diode's three-dimensional location can be computed. Given the location of three or more moving diodes, the moving body's orientation can be computed. Similar technology has been applied to the cockpit, although orientation was the only concern [13].

Figure 3: Conceptual drawing of outward-looking system and the sensors' fields of view

\section{System overview}

Wang demonstrated the viability of head-mounted lateral-effect photodiodes and overhead LEDs. This system extends his work in several ways. First, an overhead grid of 960 LEDs was produced with well-controlled LED location tolerances, and more attention was paid to controlling other error sources as well. Second, mathematical techniques were developed that allow an arbitrary number of sensors and an arbitrary number of LEDs in the field of view of each sensor to be used in the computation of head location. This resulted in an overdetermined system of equations which, when solved, was less susceptible to system error sources than the previous mathematical approach [10]. Third, the analog signals emerging from the sensors were digitally processed to reject ambient light. Finally, techniques for quickly determining the working sets of LEDs were developed.

\subsection{Sensor configuration}

Typically, optical trackers are inward-looking; sensors are fixed in the environment within which the HMD wearer moves. With Self-Tracker, Bishop and Fuchs introduced the concept of outward-looking trackers that mount the image sensors on the head, looking out at the environment (Figure 3).

If a large work area is required, outward-looking configurations have an advantage over inward-looking techniques when recovering orientation. The two are equivalent for measuring translation: moving the sensor causes the same image shift as moving the scene. Rotations are significantly different. Unless targets are mounted on antlers, an inward-looking sensor perceives a small image shift when the user performs a small head rotation. The same head rotation creates a much larger image shift with a head-mounted sensor. For a given sensor resolution, an outward-looking system is more sensitive to orientation changes.

Figure 4: Remote Processor and head unit with four sensors

To improve resolution in general, long focal lengths must be used with an optical sensor regardless of whether the configuration is inward or outward-looking. Thus, a wide-angle lens cannot significantly extend the work area of an inwardlooking system without sacrificing resolution and accuracy.

Narrow fields of view are a consequence of long focal lengths. Therefore, the HMD wearer cannot move very far before an LED leaves a given sensor's field of view. One solution is a cellular 
array of either LEDs or detectors. For an infrared system using LEDs and lateral-effect photodiodes, system cost is minimized by replicating LEDs as opposed to sensors. This is a result of both the device cost as well as the required support circuitry.

In the current system, four Hamamatsu (model S1880) sensors are mounted atop the head, as shown in Figure 4. Each sensor consists of a camera body to which a Fujinon lens (model CF 50B) is attached. The focal length of each lens is $50 \mathrm{~mm}$. Their principal points were determined experimentally by an optical laboratory. An infrared filter (Tiffen 87) is used to reject ambient light.

\subsection{Beacon configuration}

Experience with simulations and an early 48-LED prototype revealed the problem of beacon switching error: as the user moved around and the working set of beacons changed, discontinuous jumps in position and orientation occurred. These are caused by errors in the sensor locations, distortions caused by the lens and photodiode detector, and errors in the positions of the beacons in the ceiling.

To control beacon locations, we housed the LEDs in carefully constructed ceiling panels. Each $2^{\prime} \times 2^{\prime}$ panel is an anodized aluminum enclosure that encases a 20" x 20" two-sided printed circuit board. On this board are electronics to drive 32 LEDs. The LEDs are mounted in the front surface with standard plastic insets. Using standard electronic enclosure manufacturing techniques, it was relatively easy to realize an LED-to-LED centerline spacing tolerance of $.005^{\prime \prime}$ on a given panel.

The panels are hung from a Unistrut superstructure (Figure 1). At each interior vertex of a $2^{\prime}$ x $2^{\prime}$ grid, a vertically adjustable hanger mates with four panels. Four holes in the face of a panel slide onto one of four dowels on each hanger. The entire array of panels is levelled with a Spectra Physics Laser-Level, which establishes a plane of visible red light several inches below the panels' faces. Each hanger is designed to accept a sensor (Industra-Eye) that measures the vertical position of the laser relative to its own case. By moving the hangers up or down, they can be aligned to within .006" of the light beam.

The panels are electrically connected by a data and power daisy chain. The data daisy chain allows an individual LED to be selected. Once selected, the LED (Siemens SFH 487P) can be driven with a programmable current that ranges from $0-2$ amperes. The programmable current allows an electronic iris feature to be implemented. Typically, an LED will be on for no more than $200 \mu \mathrm{sec}$. During this time period, the current is adjusted to achieve a desired signal level at the sensor (see Section 4).

\subsection{Data Flow}

As shown in Figure 5, the signals emerging from the headmounted sensors are connected to the Remote Processor. Worn as a belt pack, the Remote Processor functions as a remote analog-to-digital conversion module. It can accept the four analog voltages emerging from a lateral-effect photodiode, for up to eight sensors. On command, the Remote Processor will simultaneously sample the four voltages on a selected sensor and relay four, 12-bit results to the LED Manager. The Remote Processor was used to alleviate the need for long runs of analog signals emerging from multiple sensors.
The LED Manager is a 68030-based processing module that controls the Remote Processor as well as the ceiling. A TAXIbased serial datalink [1] provides access to the Remote Processor while the ceiling's data daisy chain terminates at the LED Manager. Software executing on this module is responsible for turning LEDs on and for extracting data from the sensors. The LED Manager resides in a remote VME chassis that must be located near the ceiling structure.

\section{Figure 5: System Dataflow}

For each measurement of head location, the LED Manager produces a list of visible LEDs and their associated photocoordinates. This list is transferred via shared memory to the Collinearity module, which resides in the graphics engine's VME chassis. The i860-based Collinearity module translates the list of photocoordinates into the current estimate of head location. For reasons explained in Section 6, an additional 68030-based processor is used to aid the transfer of data from the remote system to the host. In theory, this is not required. The VME systems are connected by a Bit-3 VME buslink.

The sampled head position is communicated to the Pixel-Planes 5 graphics engine [14], which in turn updates the images on the user's displays.

\section{Low-level software}

A library of low-level routines running on the LED Manager, called the Acquisition Manager, controls the beacons and detectors. Given an LED and a photodiode unit, these routines light an LED and determine if a photodiode's detector sees that LED. The detector returns four analog signals, which the Remote Processor board digitizes. A simple formula [16] converts these four numbers into the $x, y$ photocoordinates of the LED's projection on the detector.

Hamamatsu datasheets specify 1 part in 40 accuracy and 1 part in 5000 resolution for the lateral-effect diode-based detectors used. As with Antonsson [2], we were able to achieve 
approximately 1 part in 1000 accuracy for the combined photodiode-lens assembly. Achieving this result required significant efforts to improve the signal-to-noise ratio and compensate for distortion, including:

Ambient light rejection: The voltage values with the LED off (called the "dark current") are subtracted from the voltage values with the LED on. Sampling with the LED off both before and after the samples with the LED on and averaging the two yields substantially improved ambient light rejection.

Random noise rejection: Averaging several measurements reduces random noise effects, but costs time. A good compromise between accuracy and sampling speed is to take 8 samples with the LED off, 16 samples with the LED on and 8 more samples with the LED off.

Current scaling: The distance between a photodiode and an LED depends on the user's location. To maximize the signal without saturating the photodiode detector, the Acquisition Manager dynamically adjusts the amount of current used to light an LED. Acquisition Manager routines estimate the threshold of current that will saturate the detector and use $90 \%$ of this value during sampling.

Figure 6: Optical bench for photodiode calibration

Calibration: Both the lens and the photodiode detector suffer from nonlinear distortions. By placing the photodiodes on an optical bench and carefully measuring the imaged points generated by beacons at known locations (Figure 6), we built a lookup table to compensate for these distortions. Bilinear interpolation provides complete coverage across the detector. More sophisticated calibration techniques should be investigated. Accurate calibration is required to reduce beacon switching error.

Programming techniques: Techniques such as list processing, cache management and efficient code sequencing result in a substantially improved sampling rate. In addition, expedited handling of special cases, such as when an LED is not within the field of view of a photodiode unit, further helps system performance.

Using 32 samples per LED, we compute a visible LED's photocoordinate in $660 \mu \mathrm{sec}$ and reject a non-visible LED in
100 usec. LEDs are tested in groups; each group carries an additional overhead of $60 \mu \mathrm{sec}$.

Figure 7: Sensors viewing LEDs in the ceiling. Each of the four groups is the set of LEDs that a sensor can see. Picture taken with a camera that is sensitive to infrared light.

\section{LED Manager}

The LED Manager uses the low-level Acquisition Manager routines to determine which LEDs each photodiode unit sees and where the associated imaged points are on the photodiode detectors. We usually want to collect data from all visible LEDs, since larger sample sets ultimately yield less noisy solutions from the Collinearity module (Section 7). Because the number of visible LEDs is small (see Figure 7) compared to the total number of LEDs in the ceiling, something faster than a brute-force scan of the entire ceiling array is called for. Two assumptions help us design a more efficient method:

1) Spatial coherence: The set of beacons visible to a photodiode unit in a given frame will be contiguous.

2) Temporal coherence: The user's movement rate will be slow compared to the frame rate. This implies that the field of view of a given photodiode unit does not travel very far across the ceiling between frames, so its set of visible beacons will not change much from one frame to the next.

\subsection{The basic method}

In each frame, the LED Manager goes through each photodiode unit in sequence, sampling beacons until it is satisfied that it has captured most of each photodiode unit's visible set. A basic difficulty is that we cannot be sure whether a beacon is visible or not until we attempt to sample it. The LED Manager remembers which beacons were in the camera's visible set from the previous frame. The set is called the last visible set. If the last visible set is nonempty, all beacons in that set are tested. The next action depends on how many of those beacons are actually visible:

1) All: We assume the field of view has not moved much and not many more beacons will be visible. We stop with this set and go on to the next photodiode unit.

2) Some: We assume that the field of view has shifted significantly, possibly enough to include previously unseen beacons. A shell fill (described later) is conducted, beginning with the set of beacons verified to be visible. 
3) None: The field of view has moved dramatically, gone off the edge of the ceiling, or is obscured. We check the neighbors of the last visible set. If any of these beacons are visible, they are used to start a shell fill. If none are visible, we give up on this photodiode unit until the next frame.

What if the last visible set is empty? Our course of action depends on whether we were able to compute a valid position and orientation for the head in the last frame:

1) Valid previous location: We can predict which LEDs should be visible to our photodiode unit, if the user's head is actually at the computed location, because the geometry of the head unit is known. If no LEDs are predicted to be visible, we go on to the next photodiode unit, otherwise we sample those beacons and use them as the start of a shell fill, if any of them were actually visible.

2) No valid previous location: Now we have no way to guess which beacons are visible, so we resort to a simple sweep search, which lights the beacons in the ceiling row by row, until we have tried the entire ceiling or an LED is found to be visible. In the former case, we give up, and in the latter case, we use the visible beacon as the start of a shell fill.

\subsection{Shell fill}

A shell fill starts with a set of beacons known to be visible to a sensor and sweeps outward until it has found all the beacons in the field of view.

We do this by first sampling the neighbors of the initial set of beacons. If none are found visible, the shell fill terminates, concluding that the beacons in the initial set are the only visible ones. If any are found visible, we then compute the neighbors of the beacons we just sampled, excluding those which have already been tried, and sample those. We repeat this process of sampling beacons, computing the neighbors of those found visible, and using those neighbors as the next sample set, until an iteration yields no additional visible beacons.

Assumption 1, that visible sets are contiguous, suggests that this procedure should be thorough and reasonably efficient.

\subsection{Startup}

At startup, the head location is not known and all of the last visible sets are empty. We do a sweep search, as previously described, for each photodiode unit to locate the initial visible sets.

\section{6}

\section{Communications}

Communication between the various processors in our system is done using shared memory buffers, which offer low latency and high speed. The buffers are allocated and deallocated via a FIFO queue mechanism. Data is "transmitted" when it is written to the buffer: no copying is necessary. The only communication overhead is the execution of a simple semaphore acquisition and pointer management routine. Furthermore, all processors use the same byte ordering and data type size, so no data translation is needed.
The queuing mechanism lets all modules in the system run asynchronously. LED Manager, the Collinearity module, and Pixel-Planes 5 run as fast as they can, using the most recent data in the queue or the last known data if the queue is empty.

The various processors in our system are split between two separate VME buses, which are transparently linked together by Bit-3 bus link adapters (Figure 5). A subtle bus loading problem prevents the i 860 board and the '030 board that runs LED Manager from operating in the same VME cage. This configuration increases latency because inter-bus access is significantly slower than intra-bus access, but increases throughput because the bus link allows simultaneous intra-bus activity to occur. Because the 1860 processor cannot directly access the VME bus, a second '030 board, which runs the Queue Manager, moves data between the LED Manager and the Collinearity module.

A simpler and less expensive system could be built if we acquired an i860 board that can run on the same bus as the LED Manager '030 board. This configuration would not require the Queue Manager board or the Bit-3 links and would reduce both latency and throughput.

\section{Space Resection by Collinearity}

Given the observations of beacons, we compute the position and orientation of the user's head by using a photogrammetric technique called space resection by collinearity. The basic method for a single camera is in [31]; what we describe here is our extension for using it in a multi-sensor system. Because of space limitations, the description is necessarily brief. Full details are provided in [6].

\subsection{Definitions}

Three types of coordinate systems exist: one World space (tied to the ceiling structure), one Head space (tied to the HMD), and several Photodiode spaces (one for each photodiode unit).

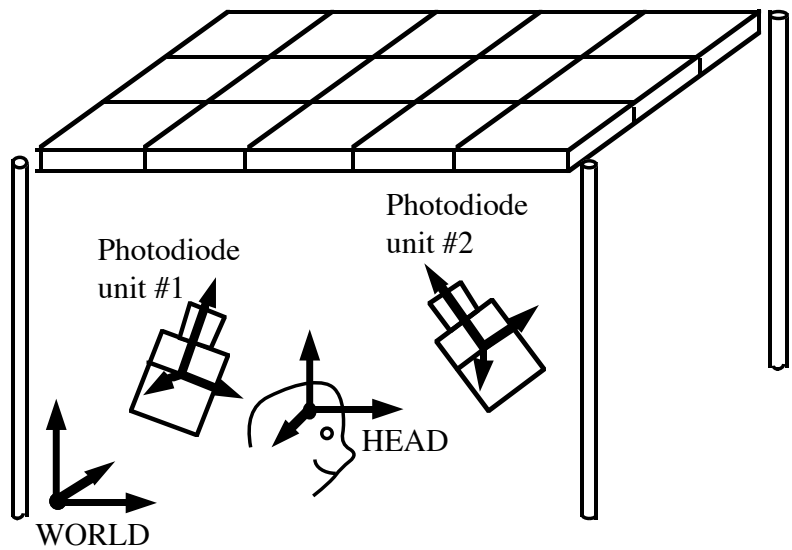

Figure 8: World, Head and Photodiode spaces

Changing representations from one space to another is done by a rotation followed by a translation. We use two types of $3 \times 3$ rotation matrices:

$\mathbf{M}=$ Head space to World space

$\mathbf{M}_{i}=$ Photodiode space $i$ to Head space 
with each matrix specified by Euler angles $\omega, \alpha$, and $\kappa$.

The optical model for each photodiode unit is simple: a light ray strikes the front principal point and leaves the rear principal point at the same angle (Figure 9).

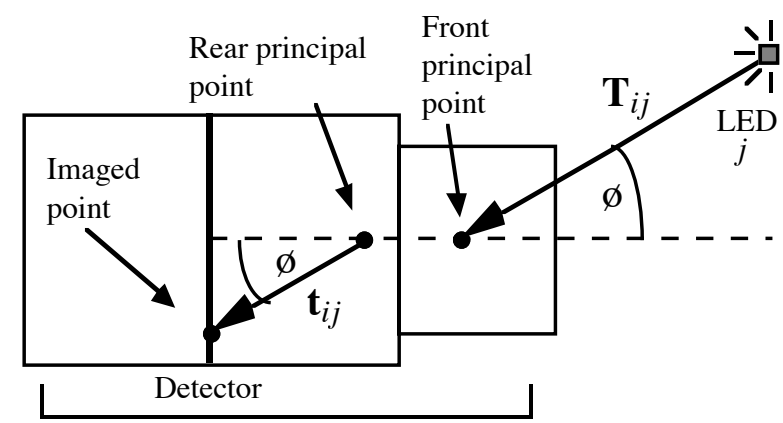

Photodiode unit $i$

Figure 9: Optical model

Finally, we list the points and vectors we will need, segregated by the coordinate system in which they are represented. Given photodiode unit $i$ sees LED number $j$,

Photodiode space:

$\left[x_{i j}, y_{i j}, 0\right]=$ imaged point on photodiode detector

Head space:

$\mathbf{t}_{i j}=$ vector from rear principal point to imaged point

$\mathbf{H}_{0}=$ origin of Head space

$\mathbf{d}_{i}=$ vector from $\mathbf{H}_{0}$ to center of photodiode detector

$\mathbf{e}_{i}=$ vector from $\mathbf{H}_{0}$ to rear principal point

$\mathbf{f}_{i}=$ vector from $\mathbf{H}_{0}$ to front principal point

World space:

$\left[X_{0}, Y_{0}, Z_{0}\right]=$ coordinates of the origin of Head space

$\left[X_{j}, Y_{j}, Z_{j}\right]=$ coordinates of LED $j$

$\mathbf{T}_{i j}=$ vector from LED $j$ to front principal point

\subsection{Geometric relationships}

Figure 9 shows that $\mathbf{T}_{i j}$ and $\mathbf{t}_{i j}$ differ only by a scale factor; if they were placed at the same start point, they would be collinear. In equations:

$$
\mathbf{T}_{i j}=\lambda \mathbf{M} \mathbf{t}_{i j}
$$

We now express $\mathbf{T}_{i j}$ and $\mathbf{t}_{i j}$ in terms of the other vectors in equations (2) and (3) and Figures 10 and 11:

$$
\begin{gathered}
\mathbf{T}_{i j}=\left[\begin{array}{c}
X_{0}-X_{j} \\
Y_{0}-Y_{j} \\
Z_{0}-Z_{j}
\end{array}\right]+\mathbf{M} \mathbf{f}_{i} \\
\mathbf{t}_{i j}=\mathbf{d}_{i}-\mathbf{e}_{i}+\mathbf{M}_{i}\left[\begin{array}{c}
x_{i j} \\
y_{i j} \\
0
\end{array}\right]
\end{gathered}
$$

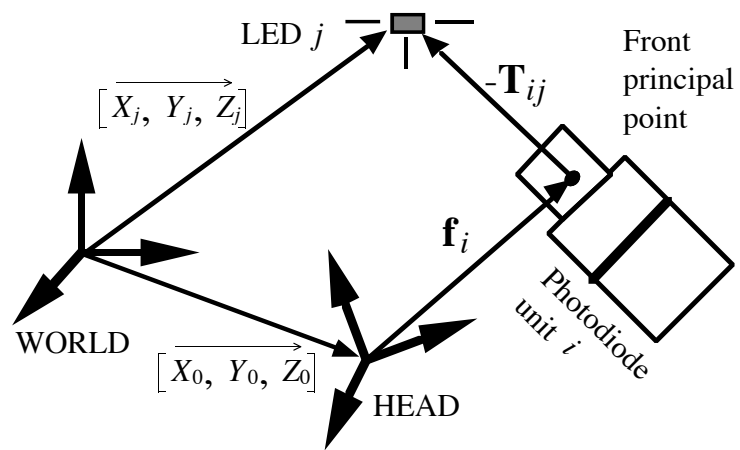

Figure 10: Expressing $\boldsymbol{T}_{i j}$ through other vectors

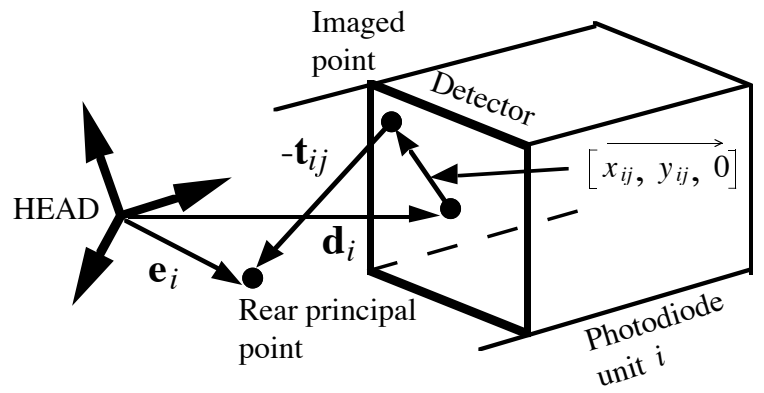

Figure 11: Expressing $\boldsymbol{t}_{i j}$ through other vectors

Substituting (2) and (3) into (1) yields the collinearity condition equation $c_{i j}$ :

$$
c_{i j}:\left[\begin{array}{c}
X_{0}-X_{j} \\
Y_{0}-Y_{j} \\
Z_{0}-Z_{j}
\end{array}\right]+\mathbf{M} \mathbf{f}_{i}=\lambda \mathbf{M}\left(\mathbf{d}_{i}-\mathbf{e}_{i}+\mathbf{M}_{i}\left[\begin{array}{c}
x_{i j} \\
y_{i j} \\
0
\end{array}\right]\right)
$$

\subsection{System of equations}

When a photodiode unit $i$ sees an LED $j$, it generates a $c_{i j}$, which represents three independent equations. If we see $N$ LEDs in all, the total number of unknowns in our system is $6+N: 3$ for position, 3 for orientation, and $N$ scale factors. The first six are what we are trying to find, but we do not care about the scale factors. We eliminate these by rearranging the $c_{i j}$ equations, then dividing the first and second equations by the third. This leaves two independent equations, of the form

$$
G 1_{i j}(\mathbf{L})=0, G 2_{i j}(\mathbf{L})=0
$$

where $\mathbf{L}$ is a vector composed of the six unknowns: position $\left(X_{0}, Y_{0}, Z_{0}\right)$ and orientation $(\omega, \alpha, \kappa$ for matrix $\mathbf{M})$. We generate a linear approximation to these two equations by applying Taylor's theorem:

$$
\begin{aligned}
-G 1_{i j}(\mathbf{L}) & =\left(\frac{\partial G 1_{i j}(\mathbf{L})}{\partial X_{0}}\right) d X_{0}+\left(\frac{\partial G 1_{i j}(\mathbf{L})}{\partial Y_{0}}\right) d Y_{0}+\left(\frac{\partial G 1_{i j}(\mathbf{L})}{\partial Z_{0}}\right) d Z_{0} \\
& +\left(\frac{\partial G 1_{i j}(\mathbf{L})}{\partial \omega}\right) d \omega+\left(\frac{\partial G 1_{i j}(\mathbf{L})}{\partial \alpha}\right) d \alpha+\left(\frac{\partial G 1_{i j}(\mathbf{L})}{\partial \kappa}\right) d \kappa
\end{aligned}
$$

and a similar expansion for the linearized $G 2$ equation.

Now we have six total unknowns, and every LED that we see generates two independent linear equations. Thus, we need to see at least three LEDs. If we see a total of $N$ LEDs, we can write 
our system of $N$ linearized $G 1$ equations and $N$ linearized $G 2$ equations in matrix form:

$$
\begin{aligned}
& -\mathbf{G}_{0}=\partial \mathbf{G} * \mathbf{D} \\
& 2 N \times 1 \quad 2 N \times 6 \quad 6 x 1
\end{aligned}
$$

where $\mathbf{D}=\left[d X_{0}, d Y_{0}, d Z_{0}, d \omega, d \alpha, d \kappa\right]^{\mathrm{T}}$,

$\partial \mathbf{G}$ is the matrix of partial derivatives of the $G 1$ and $G 2$, and $-\mathbf{G}_{0}$ contains the values of the $G 1$ and $G 2$ at a specific $\mathbf{L}$.

\subsection{Iteration and convergence}

Collinearity takes an initial guess of $\mathbf{L}$ (the unknowns) and generates correction values (in $\mathbf{D}$ ) to make a more accurate $\mathbf{L}$, iterating until it converges to a solution. Thus, we need to extract $\mathbf{D}$ from equation (4). If $N=3$, then we can solve for $\mathbf{D}$ directly. If $N>3$, then the system is overdetermined and we approximate D through singular value decomposition [24]. Simulations show that using more than the minimum of 3 LEDs can reduce average error caused by non-systematic error sources. In pseudocode, our main loop is:

Generate an initial guess for $\mathbf{L}$ repeat

Given $\mathbf{L}$, compute $\mathbf{G}_{0}$ and $\partial \mathbf{G}$

Estimate $\mathbf{D}$ using singular value decomposition $\mathbf{L}=\mathbf{L}+\mathbf{D}$

until magnitude of $\mathbf{D}$ is small return $\mathbf{L}$

How do we generate the initial guess of $\mathbf{L}$ ? Normally we use the last known position and orientation, which should be an excellent guess because we track at rates up to $100 \mathrm{~Hz}$. Collinearity usually converges in 1 or 2 iterations when the guess is close. But in degenerate cases (at system startup, or when we lose tracking because the photodiode units are pointed away from the ceiling), we have no previous $\mathbf{L}$. Collinearity will not converge if the guess is not close enough to the true value; we empirically found that being within $30^{\circ}$ and several feet of the true $\mathbf{L}$ is a good rule of thumb. So in degenerate cases, we draw initial guesses for $\mathbf{L}$ from a precomputed lookup table with 120 entries, trying them sequentially until one converges. We can double-check a result that converges by comparing the set of LEDs used to generate that solution to the theoretical set of LEDs that the photodiode units should see, if the head actually was at the location just computed. When these two sets match, we have a valid solution.

\section{Performance}

A "typical situation" is defined as a user of average height standing erect underneath the ceiling, with at least three photodiode units aimed at the ceiling, moving his head at moderate speeds. All measurement bounds assume that the user remains in tracker range with at least two sensors aimed at the ceiling.

Update rate: The update rate ranges between $20-100 \mathrm{~Hz}$. Under typical situations, $50-70 \mathrm{~Hz}$ is normal, depending on the height of the user. The wide variation in the number of LEDs seen by the sensors causes the variation in update rate. The more LEDs used, the slower the update rate, because LED Manager is the slowest step in the pipeline. If the head remains still and the sensors see a total of $B$ beacons, LED
Manager requires $3.33+0.782 * B$ ms to run. Rapidly rotating the head increases this time by a factor of about 1.33 , since additional time is required to handle the changing working sets of LEDs. Slower head movement rates have correspondingly smaller factors.

Lag: Lag varies between $20-60 \mathrm{~ms}$, with $30 \mathrm{~ms}$ being normal under typical situations. Lag is measured from the time that LED Manager starts to the time when the Collinearity module provides a computed head location to the graphics engine. Therefore, tracker latency is a function of the number of LEDs seen and the quality of the initial guess provided to the Collinearity module. As $B$ gets smaller, both the LED Manager and Collinearity modules become faster, reducing latency. This mutual dependence on $B$ means that update rate and lag are closely tied: faster update rates correspond with lower latency values.

Resolution: When moving the head unit very slowly, we observed a resolution of $2 \mathrm{~mm}$ in position and 0.2 degrees in orientation. Measuring accuracy is much harder, and we do not have any firm numbers for that yet. At SIGGRAPH '91, users were able to touch a chair and the four ceiling support poles based solely on the images they saw of models of the chair and the poles in the virtual environment.

\section{Evaluation}

The system provides adequate performance but has several limitations and problems that must be addressed. The most noticeable is the combination of excessive head-born weight and limited head rotation range. Rotation range depends heavily on the user's height and position under the ceiling. A typical maximum pitch range near the center of the ceiling is 45 degrees forward and 45 degrees back. When the user walks near an edge of the ceiling, head rotation range becomes much more restricted. To accommodate the full range of head motion, multiple image sensors must be oriented such that wherever the head is pointed, two or more sensors are able to view LEDs on the ceiling. Given the current focal lengths, simulations show that as many as eight fields of view are required for a respectable rotation range [29]. The weight of each sensor must be significantly reduced to achieve this goal.

To reduce weight, we are trying to replace the current lenses (11 oz. each) with smaller, lighter lenses (2 oz. each). Other approaches are possible. Wang proposed optically multiplexing multiple fields of view onto on a single lateraleffect photodiode [29]. Reduced signal strength, distortions, and view identification ambiguities make this a nontrivial task. It may be easier to design a helmet with integral photodiodes and lenses. Given that each photodiode is about the size of a quarter, the entire surface of a helmet could be studded with sensors.

Beacon switching error has been greatly reduced, but not eliminated. Small observable discontinuities occasionally occur, and while they are not a major disturbance, they are annoying. Calibration techniques are being explored to estimate error sources and compensate for their effects. Photogrammetric techniques like the bundle adjustment method [8] or an alternate scheme suggested by our colleagues [18] may provide the answer. 
Infrared light sources in the environment surrounding the tracker, such as sunlight or incandescent light, must be controlled for the system to operate correctly. Specifically, any light source whose wavelengths include $880 \mathrm{~nm}$ will be detected by the photodiodes as if it were an LED. For this reason, fluorescent ambient lighting is preferred. Extreme caution is not required, however. Whereas a sensor pointed directly at an infrared light source other than the LEDs will confuse the system, a certain level of indirect infrared background light is tolerable due to the combination of optical filters and the ambient light rejection techniques described in Section 4.

Surprisingly, the bottleneck in the system is the time required to extract data from the photodiode detectors, not the time required to compute the head's location. The i860 processor performs the latter task adequately, and even faster and cheaper processors will be available in the future. But getting accurate photocoordinates from the detectors takes longer than expected, because of the time spent in current scaling and in sampling multiple times per LED. Further experimentation is required to see if we can safely reduce the number of samples. Optimizing the low-level software may improve sampling speed by $20-30 \%$.

The use of Euler angles in the collinearity equations opens the possibility of gimbal lock. The current system avoids this because the head rotation range is too limited to reach gimbal lock positions, but a future version may. If we cannot place the gimbal lock positions out of reach, we can solve for the nine rotation matrix parameters individually, subject to six constraints that keep the matrix special orthogonal, or we may be able to recast the rotations as quaternions.

Since this tracker encourages the user to walk around large spaces, tripping over the supporting cables is a danger. We will investigate the feasibility of a wireless datalink to remove this problem.

Under certain circumstances, the sensors can see large numbers of beacons, such as a total of 30 or more. While using many LEDs usually improves the solution from the Collinearity module, it also slows down the update rate and increases the lag. Further experiments are needed to explore this tradeoff and determine rules of thumb that provide a reasonable balance between resolution and update rate.

Cellular systems using different technologies or configurations could be built to achieve similar scalable work areas. For example, Ascension has announced a cellular magnetic system [4]. Regardless of the technology, any cellular approach creates the problem of beacon switching error or its equivalent. Steps we took to control these errors would apply to other technologies as well: 1) precise positioning and measurement of system components, 2) averaging techniques to reduce random error sources, and 3) calibration routines to compensate for systematic error sources.

\section{Future work}

We intend to continue improving this system. In addition to the tasks listed in Section 9, we would eventually like to expand the ceiling size to around 20' $\mathrm{x} 20^{\prime}$, to provide much greater range of movement, both quantitatively and psychologically. Also, ample room exists to improve the heuristics and optimize the code, increasing the update rate and reducing latency.

But beyond these incremental improvements, we do not expect to pursue this particular technology further. The system is a vehicle for further research and provides room-sized tracking capability today for HMD applications that require it. For example, the UNC Walkthrough team has begun interviewbased user studies on what impact large-environment tracking has on the architectural design of a kitchen. In the future, emphasis will be placed on technologies that allow unlimited tracking volumes in unstructured environments. This potential exists in systems that measure only the relative differences in position and orientation as the user moves, integrating these differences over time to recover the user's location. Examples include inertial technologies and Self-Tracker. Since these technologies suffer from drift problems, initial versions may be hybrid systems reliant on the optical tracker for auxiliary information. Thus, the optical tracking system will serve as a testbed for its own successor.

Tracking HMDs will only get harder in the future. The higher resolution displays being developed demand higher resolution trackers. See-through HMDs add additional requirements. In the completely-enclosed HMDs commonly used today, the entire world is virtual, so resolution is much more important than accuracy. But for a see-through HMD, accurate registration of the HMD to the real world is vital. The effects of latency will also become more disturbing in see-through HMDs. Viewing computer-generated objects superimposed upon the real world, where those objects move with significant lag but the real world does not, will not provide a convincing illusion. People can perceive as little as $5 \mathrm{~ms}$ of lag [15], and it is unlikely that the combined tracker and graphics engine latency will be below that anytime soon. Therefore, compensation techniques need to be explored [19][24]. If HMDs are to achieve their potential of making a user truly feel immersed inside a virtual world, significant advances in tracking technologies must occur.

\section{References}

[1] Advanced Micro Devices, Am7968/Am7969 TAXIchip Article Reprints, Sunnyvale, CA.

[2] Antonsson, E. K., and R.W. Mann. Automatic 6-D.O.F. kinematic trajectory acquisition and analysis. $J$. Dynamic Systems, Measurement, and Control, 111, (March 1989) pp. 31-39.

[3] Ascension Technology Corporation. The Bird 6D Input Device, Burlington, Vermont, 1989.

[4] Ascension Technology Corporation. A Flock of Birds product description sheet, Burlington, Vermont, April 1991.

[5] Axt, Walter E. Evaluation of a pilot's line-of-sight using ultrasonic measurements and a helmet mounted display. Proceedings IEEE National Aerospace and Electronics Conf. (Dayton, OH, May 18-22, 1987) pp. 921-927. 
[6] Azuma, Ronald, and Mark Ward. Space-resection by collinearity: mathematics behind the optical ceiling head-tracker. UNC Chapel Hill Dept. of Computer Science technical report TR 91-048, Nov. 1991.

[7] Bishop, Gary and Henry Fuchs. The self-tracker: A smart optical sensor on silicon. Proceedings of the 1984 MIT Conference on Advanced Research on VLSI (Dedham, MA: Artech House, Jan 1984) pp. 65-73.

[8] Burnside, C. D. Mapping from Aerial Photographs. Granada Publishing Limited, G. Britain, 1979, pp. 248258.

[9] Chung, Jim, Mark Harris, Fred Brooks, et al. Exploring Virtual Worlds with Head-Mounted Displays. SPIE Proceedings vol. 1083 Non-Holographic True 3Dimensional Display Technologies (Los Angeles, CA, Jan 15-20, 1989).

[10] Church, Earl. Revised geometry of the aerial photograph. Bulletins on Aerial Photogrammetry, No. 15, Syracuse University, 1945.

[11] Cook, Anthony. The helmet-mounted visual system in flight simulation. Proceedings Flight simulation: Recent developments in technology and use. (Royal Aeronautical Society, London, England, Apr. 12-13, 1988) pp. 214-232.

[12] Fake Space Labs, Binocular Omni-Orientation Monitor (BOOM), Menlo Park, CA.

[13] Ferrin, Frank J. Survey of helmet tracking technologies. SPIE Vol. 1456 Large-Screen Projection, Avionic, and Helmet-Mounted Displays (1991) pp. 86-94.

[14] Fuchs, Henry, John Poulton, John Eyles, et. al. PixelPlanes 5: A Heterogeneous Multiprocessor Graphics System Using Processor-Enhanced Memories. Proceedings of SIGGRAPH '89 (Boston, MA, July 31Aug 4, 1989). In Computer Graphics 23, 3 (July 1989) pp. 79-88.

[15] Furness, Tom, and Gary Bishop. Personal communication.

[16] Hamamatsu. Hamamatsu Photonics, Hamamatsu City, Japan, 1985.

[17] Hardyman, G. M. and M. H. Smith. Helmet mounted display applications for enhanced pilot awareness. Proceedings of AIAA Flight Simulation Technologies Conference (Boston, MA, Aug. 14-16, 1989) pp. 221225.

[18] Hughes, John F., and Al Barr. Personal communication.

[19] Liang, Jiandong, Chris Shaw, Mark Green. On TemporalSpatial Realism in the Virtual Reality Environment. Proceedings of the 4th annual ACM Symposium on User Interface Software \& Technology (Hilton Head, SC, Nov 11-13 1991) pp. 19-25.

[20] Logitech, Inc. Logitech 3-D Mouse news release. July 30, 1991.

[21] POLHEMUS 3SPACE User's Manual, OPM3016-004B, Colchester, Vermont, 1987.

[22] Press, William, Brian Flannery, Saul Teukolsky, William Vetterling. Numerical Recipes in C. Cambridge University Press, USA, 1988.
[23] SELCOM. SELSPOT II HARDWARE and MULTILab Software, Southfield, Michigan, 1988.

[24] Smith Jr., B. R. Digital head tracking and position prediction for helmet mounted visual display systems. Proceedings of AIAA 22nd Aerospace Sciences Meeting, (Reno, NV, Jan. 9-12, 1984).

[25] So, Richard H., and Michael J. Griffin. Effects of time delays on head tracking performance and the benefits of lag compensation by image deflection. Proceedings of AIAA Flight Simulation Technologies Conference (New Orleans, LA, Aug. 12-14, 1991) pp. 124-130.

[26] Sorensen, Brett, Max Donath, Guo-Ben Yang, and Roland Starr. The Minnesota scanner: a prototype sensor for three-dimensional tracking of moving body segments. IEEE Transactions on Robotics and Automation, 5, 4, (August 1989), pp. 499-509.

[27] Sutherland, Ivan. A head-mounted three dimensional display. Fall Joint Computer Conference, AFIPS Conference Proceedings, 33 (1968) pp. 757-764.

[28] Wang, Jih-Fang, Vernon Chi, and Henry Fuchs. A realtime 6D optical tracker for head-mounted display systems. Proceedings of 1990 Symposium on Interactive 3D Graphics (Snowbird, Utah, 1990). In Computer Graphics 24, 2 (March 1990) pp. 205-215.

[29] Wang, Jih-Fang, Ronald Azuma, Gary Bishop, Vernon Chi, John Eyles, Henry Fuchs. Tracking a head-mounted display in a room-sized environment with head-mounted cameras. SPIE Proceedings Vol. 1290 Helmet-Mounted Displays II (Orlando, FL, Apr 19-20 1990) pp. 47-57.

[30] Welch, Brian, Ron Kruk, Jean Baribeau, et al. Flight Simulator: Wide-Field-Of-View Helmet-Mounted Infinity Display System, Air Force Human Resources Laboratory technical report AFHRL-TR-85-59, May 1986, pp. 4860 .

[31] Wolf, Paul. Elements of Photogrammetry, With Air Photo Interpretation and Remote Sensing, 2nd ed., McGraw-Hill, New York, 1983.

[32] Woltring, Herman. Single- and Dual-Axis Lateral Photodetectors of Rectangular Shape. IEEE Trans. on Electron Devices, (August 1975) pp. 581-590.

\section{Acknowledgements}

This system would not exist today without the support of the Microelectronics System Laboratory, the Graphics Laboratory staff, and the other members of the Tracker group. The authors wish to thank Gary Bishop, Vern Chi, Carney Clegg, John Eyles, David Harrison, John Hughes, Jack Kite, Mark Mine, John Poulton, C. A. Stone, John Thomas, and Norm Vogel for all of their help. We also thank Fred Brooks and the UNC Walkthrough group for providing architectural applications to use with our tracker.

Accu-Tool Corporation of Cary, NC was responsible for the head frame's design and fabrication. Panel enclosures were fabricated by Southeastern Machine Tool of Raleigh, NC. All circuit boards were fabricated by Multilayer Technologies, Inc. of Irvine, CA. This work was partially supported by ONR contract N00014-86-K-0680, DARPA contract DAEA 18-90-C0044, NSF contract ASC-8920219, and a Pogue Fellowship. 